\title{
Exchange and correlation energies of ground states of atoms and molecules in strong magnetic fields
}

\author{
P. Schmelcher ${ }^{1}$, M. V. Ivanov ${ }^{2}$ and W. Becken ${ }^{1}$ \\ 1 Theoretische Chemie, Physikalisch-Chemisches Institut, Universität Heidelberg, INF 253, D-69120 Heidelberg, Federal \\ Republic of Germany \\ ${ }^{2}$ Institute of Precambrian Geology and Geochronology, Russian Academy of Sciences, Nab. Makarova 2, St. Petersburg \\ 199034, Russia
}

\begin{abstract}
Using a Hartree-Fock mesh method and a configuration interaction approach based on a generalized Gaussian basis set we investigate the behaviour of the exchange and correlation energies of small atoms and molecules, namely the helium and lithium atom as well as the hydrogen molecule, in the presence of a magnetic field covering the regime $B=0-100 a . u$. In general the importance of the exchange energy to the binding properties of atoms or molecules increases strongly with increasing field strength. This is due to the spin-flip transitions and in particular due to the contributions of the tightly bound hydrogenic states which are involved in the corresponding ground states of different symmetries. In contrast to the exchange energy the correlation energy becomes less relevant with increasing field strength. This holds for the individual configurations constituting the ground state and for the crossovers of the global ground state.
\end{abstract}

\section{INTRODUCTION}

Particle systems in strong magnetic fields represent a source of complex nonlinear phenomena which have their origin in the competing particle-particle and magnetic interactions. Considering the simplest of all possible systems, namely the hydrogen atom [1], we encounter a variety of different properties and qualitatively new effects. Leaving aside the inherent two-body character of the system [2] let us concentrate on the evolution of the electronic properties with increasing field strength. Since the symmetry gradually changes with increasing field strength from a field-free spherical to a cylindrical one in the high field limit we encounter a strong rearrangement of the electronic wave function which is particularly dramatic in the so-called intermediate regime for which the strength of the two interactions are of comparable order of magnitude. The large number of investigations on the hydrogen atom in a magnetic field during the past twenty years provided us with a detailed understanding of the quantum properties of the atom. It thereby became a prominent example for a low-dimensional chaotic dynamical system as well as a testing ground for the development of semiclassical theories of nonintegrable systems [3].

In contrast to the hydrogen atom our knowledge on the behaviour and properties of many electron atoms in strong magnetic fields is still in its infancy. There exists a moderate number of investigations on the ground and low-lying excited states of the helium atom for field strengths ranging from the weak to the high field regime (see refs. [4] and references therein). Very recently precise calculations of a considerable number of excited states of different symmetries [8] were performed for a wide range of astrophysically relevant field strengths. As a result a successful comparison of the corresponding stationary transitions with the astronomically observed spectrum of the magnetic white dwarf GD229 could be performed resulting in a strong evidence for the existence of helium in the atmosphere of this object [9]. For atoms with more than two electrons there exist only few investigatons of partially qualitative character which focus almost exclusively on the high field regime (see, for example, ref. [10] and references therein). For the lithium atom there are two recent investigations [11, 12] which cover also the intermediate regime of field strengths.

In the case of molecules the situation is somewhat similar. The one-electron problem, i.e. the hydrogen molecular ion $H_{2}^{+}$in a magnetic field, has been studied in some detail (see refs. [13] and references therein) including the situation of an arbitrary orientation of the internuclear axis with respect to the magnetic field axis. A careful quantitative investigation of the hydrogen molecule including a correct description of the ground state for arbitrary field strengths, has however been performed only very recently (see refs. 114 16]). Nevertheless a number of interesting features have been detected like, for example, magnetic field induced chemical binding mechanisms or crossovers of ground states due to spin or internal energy shifts.

In view of the above-described dramatic changes of the electronic structure and properties of atoms and molecules in the presence of a magnetic field it is natural to ask the question how the different contributions to the total energies change with changing field strength. More precisely: how does the exchange and correlation energy behave if the field 
is turned on and increased from the weak field to the high field regime? This question is of immediate relevance to a better understanding of the binding properties of inhomogeneous electronic systems in strong fields. Furthermore our investigation is also motivated by the persisting need of density functionals in the presence of strong magnetic fields [17 19. whose construction requires detailed information about the influence of the external field on the electronic structure. The aim of the present paper is to study the behaviour of the exchange and correlation energies for a broad range of field strengths for the ground states of some selected small atoms and molecules, namely the helium and lithium atoms and the hydrogen molecule.

In detail we proceed as follows. Section 2 contains a brief discussion of relevant theoretical aspects as well as an outline of the different computational methods used to investigate the electronic structure of atoms and molecules in strong magnetic fields. Since both the theoretical background as well as the underlying computational techniques are rather sophisticated we focus here only on some major points which are of immediate relevance to the present investigation. Section 3 contains a discussion of the results, i.e. the magnetic field dependent behaviour of the exchange and correlation energies for the above-mentioned atomic and molecular two and three-electron systems. Section 4 provides a summary and the conclusions.

\section{THEORETICAL ASPECTS AND COMPUTATIONAL METHODS}

Atoms and molecules in external magnetic fields show a number of intriguing new phenomena which are not present in field-free space and which manifest themselves already on the level of the fundamental equations of motion. Examples are the inseparability of the center of mass and internal motion [2] and the screened Born-Oppenheimer approach (see ref. [20] and references therein) which has to be followed in order to ensure the validity of an adiabatic approximation for molecules in magnetic fields. In the present investigation which focuses on the ground states of the corresponding systems in the field strength regime $0<B<100$ a.u. (1a.u. corresponds to $2.35 \cdot 10^{5}$ Tesla) it can be safely assumed that the fixed-nucleus approach, i.e. the assumption of an infinitely heavy nucleus, is a very good zeroth order approximation to a more sophisticated electronic Hamiltonian. Therefore we arrive at the following general appearance of the fixed-nucleus Hamiltonian for the symmetric gauge using atomic units $(m=|e|=\hbar=1)$

$$
\mathcal{H}=\sum_{i=1}^{N}\left(\frac{\mathbf{p}_{i}^{2}}{2}+\frac{1}{2} B L_{z_{i}}+\frac{1}{8}\left(\mathbf{B} \times \mathbf{r}_{i}\right)^{2}\right)+V
$$

where we have assumed that the magnetic field is oriented along the $z$-axis. $\mathbf{r}_{i}, \mathbf{p}_{i}, L_{z_{i}}$ are the position, canonical momentum and canonical angular momentum component parallel to the field of the $i-t h$ electron, respectively. $V$ contains all the Coulomb interaction terms, i.e. the electron-nucleus attraction as well as the electron-electron and nucleus-nucleus (in the case of molecules) repulsion. In the case of an atom the symmetries of the above Hamiltonian are: rotations around the magnetic field axis, inversion of all coordinates with respect to the origin (position of the nucleus). Consequently the total angular momentum component $\sum_{i} L_{z_{i}}$ parallel to the magnetic field is conserved (with the magnetic quantum number $M$ ) and the parity and $z$-parity operator (with eigenvalue $(-1)^{\Pi_{z}}$ ) commute with $\mathcal{H}$. Including the spin multiplicity $2 S+1$ we therefore arrive at the spectroscopic notation $\nu^{2 S+1} M^{(-1)^{\Pi} z}$ of the electronic states in the presence of a magnetic field where $\nu$ designates the degree of excitation within a subspace of certain symmetries. In the case of diatomic molecules the electronic energies depend in general on both the internuclear distance as well as the angle $\Theta$ between the internuclear axis and the magnetic field axis and the symmetries of the electronic Hamiltonian depend strongly on the mutual orientation of these axes [21]. Within the present investigation we restrict ourselves to the parallel configuration $\left(\Theta=0^{\circ}\right)$ of the hydrogen molecule which has been shown to be of particular importance for the ground state of the diatomic system [21, 16, . Choosing as the origin of the internal coordinate system the midpoint between the positions of the identical nuclei we again have the parity, $z-$ parity and rotations around the magnetic field axis as symmetries and use correspondingly the molecular spectroscopic notation $\nu^{2 S+1} M_{u / g}$ where $u / g$ denotes the ungerade-gerade parity, respectively, and $M=0,1,2, \ldots$ equals the series $\Sigma, \Pi, \Delta, \ldots$

Solving the Schrödinger equation belonging to the Hamiltonian (1) in particular in the intermediate regime of field strengths poses a hard methodological and computational problem. This is due to the fact that the diamagnetic and Coulomb interaction terms are of qualitatively different nature and yield already on the one particle level (one-electron atom or molecule) a nonseparable problem. Therefore the well-established methods for ab initio calculations of atoms and molecules cannot be directly applied to electronic structure calculations in a strong magnetic field. Within the present investigation we use two methods developed during the past years especially for the situation of strong fields. They have been proven to be very accurate and efficient for studies of electronic systems in external fields in general. These are first an unrestricted Hartree-Fock (HF) approach based on a numerical mesh method and second a configuration interaction approach based on a generalized anisotropic Gaussian basis set. Combining the results of 
the corresponding calculations we will be able to extract the desired information on the behaviour of the exchange and correlation energies for small atomic and molecular systems with changing field strength. In the following we provide some key ideas and features of the above-mentioned two methods. For further details and technical aspects we refer the reader to the corresponding literature given below.

Our unrestricted HF approach is formulated in cylindrical coordinates $(\rho, \phi, z)$. We assign to each electron a certain value of the magnetic quantum number $m_{\mu}$ and each single-electron wave function $\Psi_{\mu}$ depends on the variables $\phi$ and $(\rho, z)$ according to

$$
\Psi_{\mu}(\rho, \phi, z)=(2 \pi)^{-1 / 2} e^{-i m_{\mu} \phi} \psi_{\mu}(z, \rho)
$$

where $\mu$ indicates the numbering of the electrons. The resulting partial differential equations for $\psi_{\mu}(z, \rho)$ and the formulae for the Coulomb and exchange potentials are given in ref. [7]. The one-particle equations for the wave functions $\psi_{\mu}(z, \rho)$ are solved by means of the numerical mesh method described in refs. [22,]]. The feature which distinguishes the present calculations from those described in ref. [7] is the computational technique for the evaluation of the Coulomb and exchange integrals. Here as well as in ref. [23] we obtain these potentials as solutions of the corresponding Poisson equations. The problem of the boundary conditions for the Poisson equation as well as the problem of simultaneously solving Poissons equations and Schrödinger-like equations for the wave functions $\psi_{\mu}(z, \rho)$ on the same meshes have been discussed in ref. [7]. We solve these problems by using special forms of non-uniform meshes. Solutions to the Poisson equation on separate meshes contain some errors $\delta_{P}$ associated with an inaccurate description of the potential far from the nucleus. However due to the special form of the function $\delta_{P}(h)$ for these meshes (where $h$ is a formal mesh step) the errors do not show up in the final results for the energy and other physical quantities, which we obtain by means of the Richardson extrapolation procedure (polynomial extrapolation to $h=0$ [22,24]). An additional improvement with respect to the precision of our numerical calculations of the integrals is achieved by solving the Poisson equation not for the whole charge distribution but for the total distribution minus some properly chosen charge distribution with known analytical solution to the Poisson equation.

Our mesh approach is flexible enough to yield precise results for arbitrary field strengths. Some minor decrease of the precision appears in very strong magnetic fields. This phenomenon is due to a growing difference in the binding energies $\epsilon_{B \mu}$ of single-electron wave functions belonging to the same electronic configuration

$$
\epsilon_{B \mu}=\left(m_{\mu}+\left|m_{\mu}\right|+2 s_{z \mu}+1\right) \gamma / 2-\epsilon_{\mu}
$$

where $\epsilon_{\mu}$ is the single-electron energy and $s_{z \mu}$ is the spin $z$-projection. This results in big differences with respect to the spatial extension of the density distribution for different electrons. The precision of our HF results depends, of course, on the number of mesh nodes and can be improved in calculations with denser meshes. The absolute accuracy of the total energies, i.e. the error within our HF mesh approach of the total energies, is typically of the order of magnitude of $10^{-6}$, i.e they are very well converged.

Our second method for electronic structure calculations in strong magnetic fields is a configuration interaction (CI) approach based on an atomic basis set of generalized anisotropic Gaussian orbitals (GAGO). It has originally been developed and implemented for molecules [25, 15, 16] and very recently has been specialized and optimized in order to perform electronic structure calculations of atomic systems [8]. In contrast to the above HF-approach this method is exact in the sense that it does not miss the correlation energy: increasing the number of basis functions allows, in principle, for an arbitrary accurate description of the electronic wave functions. Together with the above HF-energies this enables us to extract the behaviour of the correlation energy as a function of the field strength.

In the remaining part of this section we briefly describe the essential strategy of our GAGO based CI approach. For the spatial part of the electronic eigenfunctions of the Hamiltonian (1) we use the linear combination of atomic orbitals ansatz, i.e. we decompose the wave function into orbital configurations which respect the corresponding symmetries and the Pauli principle. In the case of the hydrogen molecule $\mathrm{H}_{2}^{+}$molecular orbital are formed as an intermediate step. The $\mathrm{H}_{2}^{+}$molecular orbitals are built from atomic orbitals centered at each nucleus. As already mentioned a key ingredient of this procedure is the GAGO basis set. The individual orbitals have to be optimized in the presence of a magnetic field in order to ensure an efficient and accurate description of the atomic or molecular properties. The nonlinear optimization of the corresponding variational exponential parameters has to be performed for each state of a given symmetry and for each field strength separately and represents therefore a very extensive work. Finally in order to determine the atomic or molecular electronic wave functions we use the variational principle which means we solve the resulting generalized eigenvalue problem via standard methods. It is very well-known that describing the correlation behaviour accurately is a very hard task to accomplish. Within the present approach the relative accuracy of the total energies is estimated to be between $10^{-3}$ and $10^{-5}$ and the errors of the determined correlation energies (using the corresponding HF results) are at most a few percent. 


\section{RESULTS AND DISCUSSION}

In the present section we will first focus on the behaviour of the exchange energies and subsequently correlation energies as a function of the magnetic field strength for atoms and thereafter for molecules. We will provide the absolute values of these energies for a grid of values of the field strength in the regime $B=0-100$ and furthermore, as an indicator of the relevance of these energies to the binding energies, we will show the ratios of these energies and the total binding energies as a function of the field strength.

Let us begin with the neutral two-electron atomic system, i.e. the helium atom. Covering the regime of field strengths $B=0-100$ the helium atom possesses ground states of different symmetries. For the regime of field strengths $0<B<0.7$ the ground state is the $1^{1} 0^{+}$state which evolves continuously from the ground state in the absence of a magnetic field. At $B \approx 0.7$ there occurs a crossover and the new ground state is of $1^{3}(-1)^{+}$symmetry which remains the ground state for the complementary regime $B>0.7$, i.e. for arbitrary high magnetic field strengths. Since the spin singlet $1^{1} 0^{+}$state possesses no exchange energy we are exclusively interested in the triplet state $1^{3}(-1)^{+}$. Table I gives the absolute values of the exchange energy $E_{e x}$ of the $1^{3}(-1)^{+}$state for twenty values of the field strength in the regime $B=0-100$. We observe a monotonous increase from $E_{e x}=0.00644$ for $B=0$ to $E_{\text {ex }}=0.43872$ for $B=100$ i.e. by approximately two orders of magnitude. This clearly demonstrates there is a strong increase in the absolute value of the exchange energy with increasing field strength. Since however the binding energy of the helium atom in the $1^{3}(-1)^{+}$state raises also with increasing field strength we have to consider the ratio $R_{x b}$ of the exchange energy and the total binding energy in order to obtain an indicator for the influence of the exchange energy on the binding properties of the atom with changing field strength. This is illustrated in Figure 1 where the behaviour of $R_{x b}$ for the $1^{3}(-1)^{+}$state of the helium atom is shown for $10^{-3}<B<10^{2}$ on a double logarithmic scale. $R_{x b}$ increases by more than one order of magnitude showing that the importance of the exchange energy for the binding properties of the $1^{3}(-1)^{+}$state of the atom increases with increasing field strength.

What is the origin of this dramatic change in the exchange energy with increasing field strength? To elucidate this we have to take a step back and consider the hydrogen atom in a magnetic field. It is well-known that each of the lowest electronic states of gerade z-parity and a given (negative) magnetic quantum number shows a monotonous increase in binding energy with increasing field strength [26]. These states uniquely correspond to the series of field-free hydrogen states $1 s_{0}, 2 p_{-1}, 3 d_{-2}, \ldots$. Indeed their binding energy diverges like $E_{B} \rightarrow \infty$ in the mathematical limit $B \rightarrow \infty$. At the same time the expectation value of the radius for these orbitals goes to zero, i.e. $<r>\rightarrow 0$. Moreover, the binding energies of all these orbitals have small differences in the limit $B \rightarrow \infty$. As a result their expectation values $<r>$ are also close together and this means that the electron densities corresponding to these orbitals are concentrated in the same spatial region. On the other hand the binding energies and the spatial electron density distributions for these states are very different for $B=0$. The $1^{3}(-1)^{+}$state of helium consists, in the language of HF, of the $1 s 2 p-1$ orbitals constituting, according to the above, two strongly bound orbitals which shrink monotonically with increasing field strength. Comparing for different field strengths the relative changes of $<r>$ for the two orbitals $1 s$ and $2 p-1$ it is obvious that they are much larger for the $2 p_{-1}$ orbital. In the limit $B \rightarrow \infty$ this orbital overlaps strongly with the $1 s$ orbital. This causes a strong increase in the overlap of the orbitals involved in the integral expression for the exchange energy and thereby explains the above-observed increase of the exchange energy with increasing field strength.

Next let us consider the neutral three-electron atom, i.e. the lithium atom in a magnetic field [12]. The field-free ground state possesses ${ }^{2} 0^{+}$symmetry and, in a HF language, is represented by the spin dublet configuration $1 s^{2} 2 s$. The binding energy of this state increases monotonically with increasing field strength. A careful investigation shows [12] that there exist two crossovers with respect to the ground state of the Li atom with increasing field strength finally ending up in a spin quartett state. The first crossover occurs at $B_{c 1}=0.176$ up to which the $1^{2} 0^{+}$remains the global ground state. The new ground state above $B_{c 1}$ is constituted by the $1^{2}(-1)^{+}$state which, in the HF picture, is represented by the spin dublet configuration $1 s^{2} 2 p_{-1}$. This first change in the ground state is therefore not associated with a spin flip process but a pure change in the spatial orbitals: the weakly bound $2 s$ orbital is replaced by the strongly bound $2 p_{-1}$ orbital. The $1^{2}(-1)^{+}$spin dublet state is the global ground state of the Li atom in the regime $0.176<B<2.153$. At $B_{c 2}=2.153$ a second crossover takes place and for $B>B_{c 2}$ the new ground state is represented by the fully spin polarized $1^{4}(-3)^{+}$state which is constituted by the $1 s 2 p_{-1} 3 d_{-2}$ orbital configuration.

Table 1 provides the exchange energies of the above-mentioned ground states $1^{2} 0^{+}, 1^{2}(-1)^{+}$and $1^{4}(-3)^{+}$of the lithium atom, respectively, for the same grid of field strengths used for the helium atom. Starting with a vanishing field strength the exchange energy for the 'weak-field ground state' $1^{2} 0^{+}$first increases with increasing field strength by approximately a factor two up to $B \approx 1$ and subsequently decreases finally $(B=100)$ being lower than the original field-free value. A similar behaviour can be observed for the corresponding quantity $R_{x b}(B)$ the drop after the increase being however even larger since the total binding energy increases with increasing field strength. The exchange energies of the $1^{2}(-1)^{+}$and $1^{4}(-3)^{+}$states of the lithium atom increase monotonically with increasing field 
strength in the complete regime $0<B<100$. The factor of increase is approximately 40 for the $1^{4}(-3)^{+}$state and even larger, i.e. 200 , for the $1^{2}(-1)^{+}$state. Analogously the quantity $R_{x b}$ increases with increasing field strength for both states (see figure 1). The latter clearly indicates the increasing importance of the exchange energy for the binding properties of the atom with increasing field strength. The explanation provided above for the increase of the exchange energy of the helium atom in a magnetic field holds also for the $1^{2}(-1)^{+}$and $1^{4}(-3)^{+}$states of lithium. The HF-orbitals responsible for the increase of the exchange energy are the $1 s, 2 p$ and $1 s, 2 p, 3 d$ orbitals, respectively. Obviously this argument does not hold for the $1^{2} 0^{+}$state since the involved $2 s$ HF-orbital shows no unrestricted shrinking in size with increasing field strength.

We now investigate the correlation energies for the ground states $1^{1} 0^{+}$and $1^{3}(-1)^{+}$of the helium atom. (Unfortunately there exists no approach currently which would allow to investigate the correlation energy of more than two-electron systems in the presence of a strong magnetic field). The corresponding values are given in table 2 for a grid of eleven field strengths in the regime $0<B<100$. Let us first consider the 'weak-field ground state' $1^{1} 0^{+}$. Its correlation energy stays approximately constant up to a field strength of 2 and then increases only by a factor of two while increasing the field strength up to 100 . Figure 2 shows the ratio $R_{c b}$ of the correlation energy and the corresponding total binding energy. $R_{c b}$ stays approximately constant up to $B=0.1$ and decreases thereafter which indicates that the correlation energy becomes increasingly unimportant for the binding properties of the $1^{1} 0^{+}$state with increasing field strength. If we focus on the correlation energies of the $1^{3}(-1)^{+}$state of the helium atom we observe (see table 2) a monotonous increase of roughly one order of magnitude for the whole regime $B=0-100$. $R_{c b}$ for the $1^{3}(-1)^{+}$state first increases (see figure 2 ) with increasing field strength up to $B \approx 1$, then shows a minor decrease and finally increases again for $B_{\sim}^{>10}$. The absolute values of the correlation energies of this state are, for any field strength, much smaller than the corresponding exchange energies. The observed relative changes of the correlation energies with increasing field strength are also significantly smaller than those observed for the exchange energies. One can therefore conclude that the importance of the correlation energy for the binding properties of the 'weak-field ground state' $\left(1^{1} 0^{+}\right)$of helium decreases with increasing field strength and that the exchange energy in case of the 'high-field ground state' $\left(1^{3}(-1)^{+}\right)$of helium becomes increasingly important for the binding properties whereas its correlation energy shows only some minor fluctuations.

We now turn to a discussion of the exchange and correlation energies for the ground states of the hydrogen molecule in the presence of a magnetic field which is oriented parallel to the internuclear axis. Refs. 14 16. very recently clarified the question of the global ground states of the molecule for arbitrary field strengths. In field free space the ground state is well-bound and of ${ }^{1} \Sigma_{g}^{+}$symmetry, i.e. in particular a spin singlet state. In the presence of a magnetic field the spin-Zeeman shift lowers the total energy for triplet states with $M_{s}=-1$. As a result a ground state crossover between the potential energy curves of the singlet $1^{1} \Sigma_{g}$ and triplet $1^{3} \Sigma_{u}$ state occurs at $B \approx 0.18$. For $B_{c_{1}}>0.18$ the minimum of the potential energy curve of the $1^{1} \Sigma_{g}$ state lies higher in energy than the dissociation limit of the essentially repulsive (see below) potential energy curve of the $1^{3} \Sigma_{u}$ state. The latter represents therefore the global ground state of the hydrogen molecule if we increase the field strength beyond $B_{c_{1}}$. The potential energy curve of the $1^{3} \Sigma_{u}$ state is a predominantly repulsive curve for any field strength. Only for $B_{\sim}^{<} 1.0$ it exhibits a shallow outer van der Waals well whose depth is of the order to $10^{-5}$ and contains no vibrational levels. The hydrogen molecule is therefore unstable in the regime of field strengths $B_{c_{1}}<B<B_{c_{2}}$ where $B_{c_{2}}=12.3$ is the field strength for which a second crossover takes place. The new ground state above $B_{c_{2}}$ is the state $1^{3} \Pi_{u}$ which is a strongly bound state with a monotonically increasing binding energy with increasing field strength.

Due to antiparallel spins the 'weak-field ground state' $1^{1} \Sigma_{g}$ possesses a vanishing exchange energy. The $1^{3} \Sigma_{u}$ state is also of little interest since it exhibits only a very shallow outer well and does not correspond to a physically bound state. Nevertheless we have provided in table 3 the positions of the outer minimum as well as the exchange energies for the $1^{3} \Sigma_{u}$ state for field strengths $B \leq 1.0$. The observed changes in the exchange energy are very minor though the absolute values are pretty large. The latter fact has its origin in the exchange effects occuring because the inner part of the wave function is very similar to the corresponding wave function of the hydrogen molecular ion which is very different from the dissociative wave function of separate atoms. Of much more interest is the 'high-field ground state' $1^{3} \Pi_{u}$ whose equilibrium internuclear distances and exchange energies are given in table $3 . R_{e q}$ decreases whereas the exchange energy increases by a factor of 20 with increasing field strength $B=0-100$. Figure 3 shows the quantity $R_{x b}$ for the $1^{3} \Pi_{u}$ state. It is almost constant for $10^{-3}<B<10^{-1}$ and increases then approximately by a factor of two within the next two orders of magnitude of the field strength. Thereafter, i.e. for $B \gtrsim 10.0$, it shows only a minor decrease. The origin of this observed increase of the exchange energy of the $1^{3} \Pi_{u}$ state is very similar to the one observed for the 'high field ground states' of helium and lithium. In a HF-picture the $1^{3} \Pi_{u}$ state is described by the united-atom orbitals $1 s 2 p_{-1}$ which show a monotonically increasing binding energy and decreasing size with increasing field strength. Consequently the size of the molecule decreases also and its exchange energy increases due to the increasing overlap of the orbitals (see above). We remark that showing instead of the quantity $R_{x b}(B)$ the ratio $R_{x d}(B)$ of the exchange energy and the dissociation energy for the $1^{3} \Pi_{u}$ state would have resulted first in a moderate increase (factor of 2.5 ) with increasing field strength with a maximum at approximately $B \approx 2.0$ followed 
by a moderate decrease with further increasing strength of the field.

Finally we turn to a discussion of the correlation energies of the three states involved in the ground states of the hydrogen molecule in a magnetic field. For the 'weak-field ground state' $1^{1} \Sigma_{g}$ the correlation energy increases by approximately a factor of three with increasing field strength $B=0-100$. Figure 4 shows the corresponding ratio $R_{c b}(B)$ which is almost constant in the regime $10^{-3}<B<10^{-1}$ and then decreases monotonically with increasing field strength. The corresponding quantity $R_{c d}(B)$ which describes the ratio of the correlation energy and the dissociation energy shows a very similar behaviour. The $1^{3} \Sigma_{u}$ state shows, according to table 4 , an extremely small contribution of the correlation energy which is almost of the same size as our computational error. More interesting is again the correlation energy of the $1^{3} \Pi_{u}$ state which decreases (see table 4 ) monotonically by one order of magnitude in the complete range $B=0-100$. Apart from some small amplitude oscillations the corresponding quantity $R_{c b}(B)$ is constant. Again the ratio $R_{c d}(B)$ shows a similar behaviour, i.e. is also approximately constant.

\section{SUMMARY AND CONCLUSIONS}

We have investigated the behaviour of the exchange and correlation energies for small atoms and molecules in magnetic fields covering the regime $B=0-100$. To get the exchange energies Hartree-Fock calculations have been performed for the helium and lithium atoms and for the hydrogen molecule using a flexible mesh approach in order to adapt to the changing symmetries in the presence of the external field. To extract the correlation energies we used a configuration interaction approach which is based on an anisotropic generalized Gaussian basis set particularly developed for the needs in a strong magnetic field. Correlation energies have been determined for the helium atom and the hydrogen molecule.

The overall tendency of the exchange energy with increasing field strength is twofold. If less than two orbitals with parallel spins of the hydrogenic series $1 s, 2 p_{-1}, 3 d_{-2}, \ldots$ are involved in the Hartree-Fock wave function then it can be expected that the exchange energy does not increase significantly with increasing field strength and its relevance with respect to the binding energy can be expected to decrease. An example for this situation is given by the ground state of the lithium atom for weak magnetic fields, i.e. the $1^{2} 0^{+}$state which is constituted by the $1 s^{2} 2 s$ HF-configuration. In ref. [12] it has been shown that, with increasing field strength, the HF-configurations of the global ground states of an atom show an increasing degree of spin polarization and, more important, involve more and more of the orbitals belonging to the tightly bound hydrogenic orbitals $1 s, 2 p_{-1}, 3 d_{-2}, \ldots$. The generic behaviour of the exchange energy is then given by a monotonous, in many cases strong, increase with increasing field strength. In fact the exchange energy increases for a typical case even faster than the binding energy and becomes therefore increasingly relevant for the binding properties of the atom. Examples for this behaviour are given by the $1^{3}(-1)^{+}$ state of helium constituted by the $1 s 2 p_{-1}$ HF-configuration and the $1^{2}(-1)^{+}$and $1^{4}(-3)^{+}$states of lithium which correspond to the HF-configurations $1 s^{2} 2 p_{-1}$ and $1 s 2 p_{-1} 3 d_{-2}$, respectively. For the hydrogen molecule the strongly bound ground state in the high-field regime, i.e. the $1^{3} \Pi_{u}$ state, shows such a behaviour, although it is not as well pronounced as in the case of the above-mentioned states of helium and lithium.

So far we have summarized only the behaviour of the exchange energy for a given electronic state. However, to get the complete picture one should take into account also the fact that the ground state changes its symmetry with changing field strength. The magnetic field increasingly spin-polarizes atoms and molecules with increasing magnetic field strength, i.e. there occurs a series of ground state crossovers which are due to spin flip transitions (note that these are not the only ground state crossovers which actually occur but we have also crossovers without spin flip transitions like for example the $1^{2} 0^{+} \rightarrow 1^{2}(-1)^{+}$crossover in the case of the lithium atom). With each of those spin-flip transitions the number of unpaired electrons of the global ground state increases and gives a good chance to increase the corresponding exchange energy of the global ground state in an abrupt way. This mechanism adds to the increasing importance of the exchange energy for a given electronic state of a certain symmetry with increasing field strength. In total one can therefore make the general statement that with increasing field strength the exchange energy becomes increasingly relevant for the binding properties of atoms and molecules.

For the correlation energy the situation is very different, indeed, it is in a sense almost the opposite. Let us first discuss again the behaviour for an electronic ground state of a given symmetry. The absolute value of the correlation energy increases in most cases with increasing field strength $B=0-100$ only by a minor factor $1-10$ which is much less than the corresponding increase in exchange energy (c.f. the ground states of helium or the hydrogen molecule). Consequently, since in most cases the binding energy increases with increasing field strength, the ratio of the correlation energy and the total binding energy decreases or stays approximately constant. This means that the correlation energy becomes either increasingly irrelevant or at least does not become more relevant if we turn on a stronger and stronger magnetic field. If we consider now the sequence of global ground states with different symmetries which occurs with increasing field strength then the absolute value of the correlation energy drops at 
each crossover which involves more of the tightly bound orbitals of the hydrogenic series $1 s, 2 p_{-1}, 3 d_{-2}, \ldots$. This feature adds to the decreasing importance of the correlation energy for a given electronic ground state with a certain symmetry and increasing field strength. One can therefore conclude that with increasing field strength the correlation energy becomes increasingly irrelevant for the binding properties of atoms and molecules.

It is evident that there might be exceptions to the above-drawn picture which is concluded from the few data available on small atoms and molecules. Further investigations have to confirm and/or extend these conclusions. However this would take a considerable effort in particular with respect to the development of exact methods for more than two-electron systems which are currently not available.

\section{Acknowledgements}

This work was supported by the National Science Foundation through a grant (P.S.) for the Institute for Theoretical Atomic and Molecular Physics at Harvard University and Smithsonian Astrophysical Observatory. M.V.I. and W.B. acknowledge financial support from the Deutsche Forschungsgemeinschaft.

[1] H.Friedrich and D.Wintgen, Phys.Rep. 183, 37 (1989)

[2] P.Schmelcher and L.S.Cederbaum, 'Atoms and Molecules in Intense Fields' Springer Series: Structure and Bonding 86, 27 (1997)

[3] J.Main, 'Classical, semiclassical and quantum dynamics in atoms' edited by H.Friedrich and B.Eckardt, Springer Verlag 1997, 248

[4] H.Ruder, G.Wunner, H.Herold and F.Geyer, 'Atoms in Strong Magnetic Fields', Springer Verlag A-A (1994)

[5] M.D.Jones, G.Ortiz and D.M.Ceperley, Phys.Rev.E55, 6202 (1997)

[6] M.Braun, W.Schweizer and H.Elster, Phys.Rev.A57, 3739 (1998)

[7] M.V.Ivanov, J.Phys.B 27, 4513 (1994)

[8] W.Becken, P.Schmelcher and F.K.Diakonos, subm. to PRA

[9] S.Jordan, P.Schmelcher, W.Becken and W.Schweizer, Astron. and Astrophys. 336, L33-L36 (1998)

[10] M.C.Miller and D.Neuhauser, Mon.Not.Roy.Astr.Soc. 253, 107 (1991)

[11] M.D.Jones, G.Ortiz and D.M.Ceperley, Phys.Rev.A 54, 219 (1996)

[12] M.V.Ivanov and P.Schmelcher, Phys.Rev.A 57, 3793 (1998)

[13] U.Kappes and P.Schmelcher, Phys.Rev.A 51, 4542 (1995); Phys.Lett.A 210, 409 (1996); Phys.Rev.A 53, 3869 (1996); Phys.Rev.A 54, 1313 (1996)

[14] Yu.P.Kravchenko and M.A.Liberman, Phys.Rev.A 56, R2510 (1997); Phys.Rev.A 573403 (1998)

[15] T.Detmer, P.Schmelcher, F.K.Diakonos and L.S.Cederbaum, Phys.Rev.A 56, 1825 (1997)

[16] T.Detmer, P.Schmelcher and L.S.Cederbaum, Phys.Rev.A 57, 1767 (1998)

[17] G.Vignale and M.Rasolt, Phys.Rev.B 3710685 (1988)

[18] P.Skudlarski and G.Vignale, Phys.Rev.B 48, 8547 (1993)

[19] A.Holas and N.H.March, Phys.Rev.A 56, 4595 (1997)

[20] P.Schmelcher, L.S.Cederbaum and U.Kappes, Kluwer Academic Publishers, Conceptual Trends in Quantum Chemistry, 1 (1994)

[21] P.Schmelcher and L.S.Cederbaum, Phys.Rev.A41, 4936 (1990)

[22] M.V.Ivanov, J.Phys.B 21, 447 (1988)

[23] M.V.Ivanov, Phys.Lett.A 239, 72 (1998)

[24] M.V.Ivanov, USSR Comput.Math.\& Math.Phys. 26, 140 (1986)

[25] P.Schmelcher and L.S.Cederbaum, Phys.Rev.A 37, 672 (1988)

[26] J.E.Avron, I.W.Herbst and B.Simon, Phys.Rev.A20, 2287 (1979) 


\section{Figure Captions}

Figure 1: The ratio $R_{x b}$ of the exchange energy and the total binding energy as a function of the field strength $B$ on a double logarithmic scale: for the high field ground state of helium ${ }^{3}(-1)^{+}$(solid line) and the three electronic states of lithium ${ }^{2} 0^{+}$(dashed line), ${ }^{2}(-1)^{+}$(dotted line) and ${ }^{4}(-3)^{+}$(dash dotted line) which form the ground states for arbitrary field strengths $\left(B=1\right.$ a.u. corresponds to $2.35 \times 10^{5}$ Tesla $)$.

Figure 2: The ratio $R_{c b}$ of the correlation energy and the total binding energy as a function of the field strength $B$ on a double logarithmic scale: for the two states ${ }^{1} 0^{+}$(solid line) and ${ }^{3}(-1)^{+}$(dashed line) of helium forming the ground states for arbitrary field strengths.

Figure 3: The ratio $R_{x b}$ of the exchange energy and the total binding energy as a function of the field strength $B$ on a double logarithmic scale for the high field ground state ${ }^{3} \Pi_{u}$ of the hydrogen molecule.

Figure 4: The ratio $R_{c b}$ of the correlation energy and the total binding energy as a function of the field strength $B$ on a double logarithmic scale: for the electronic states ${ }^{1} \Sigma_{g}$ (solid line) and ${ }^{3} \Pi_{u}$ (dashed line) of the hydrogen molecule.

\section{Table Captions}

Table 1: The exchange energies of the ${ }^{3}(-1)^{+}$state of helium and the three electronic states ${ }^{2} 0^{+},{ }^{2}(-1)^{+}$and ${ }^{4}(-3)^{+}$of lithium for field strengths in the range $B=0-100$. All quantities are given in atomic units.

Table 2: The correlation energies of the ${ }^{1} 0^{+}$and ${ }^{3}(-1)^{+}$states of helium for field strengths in the range $B=0-100$. All quantities are given in atomic units.

Table 3: The exchange energies of the electronic ${ }^{3} \Sigma_{u}$ and ${ }^{3} \Pi_{u}$ states of the hydrogen molecule at the corresponding equilibrium internuclear distances $R_{e q}$ for field strengths in the range $B=0-100$. All quantities are given in atomic units.

Table 4: The correlation energies of the electronic ${ }^{1} \Sigma_{g},{ }^{3} \Sigma_{u}$ and ${ }^{3} \Pi_{u}$ states of the hydrogen molecule at the corresponding equilibrium internuclear distances $R_{e q}$ for field strengths in the range $B=0-100$. All quantities are given in atomic units. 
Figure 1

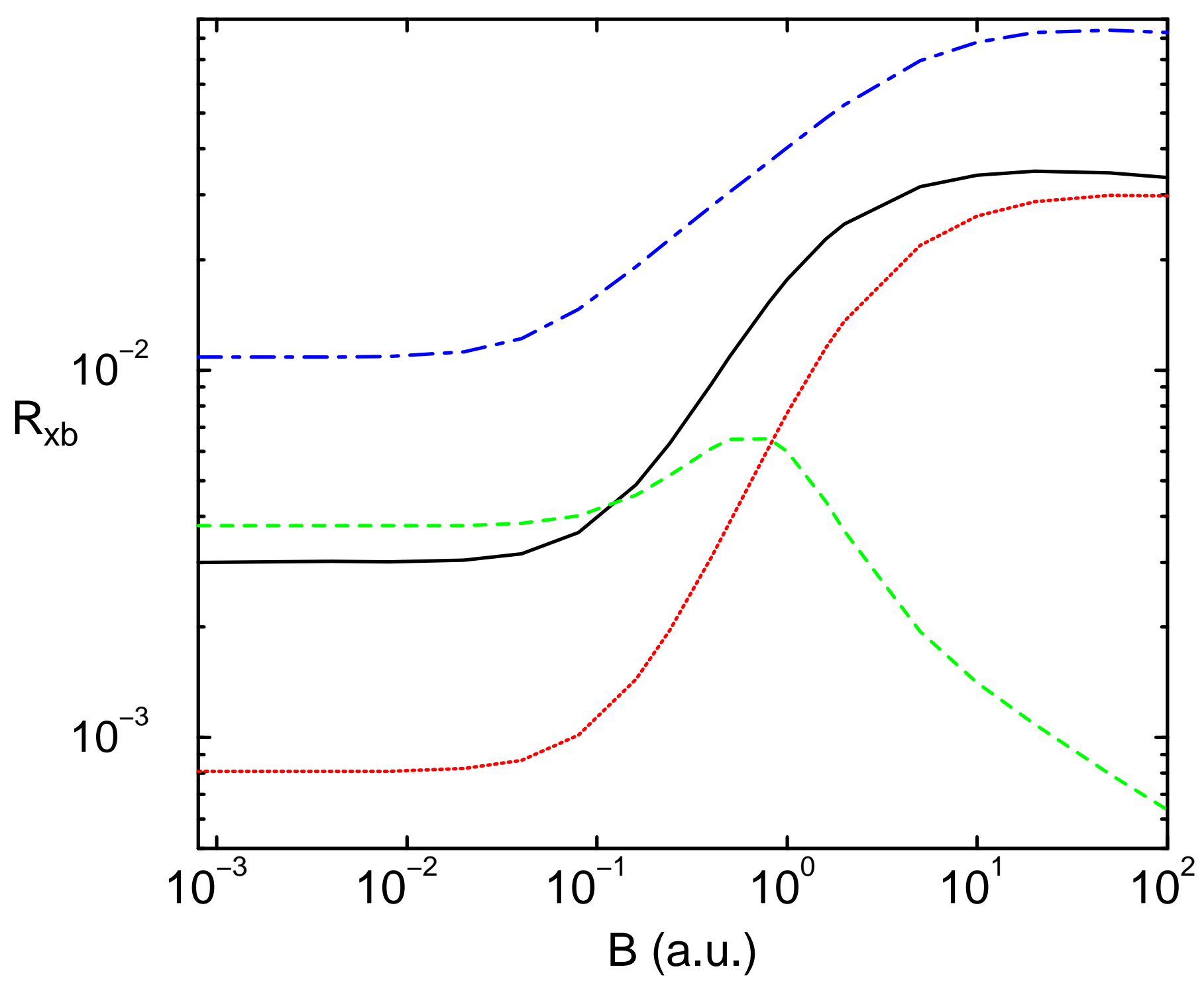


Figure 2

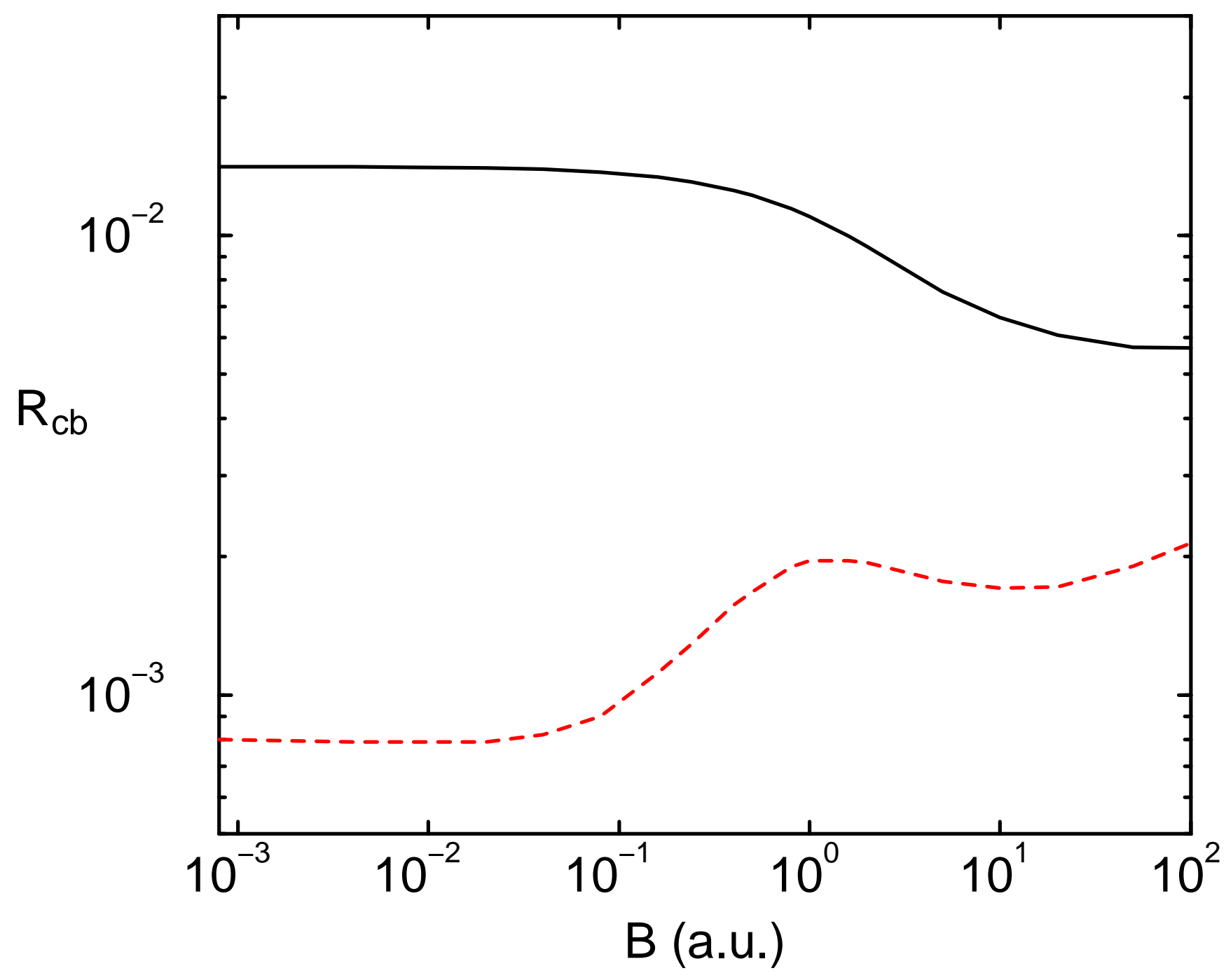


Figure 3

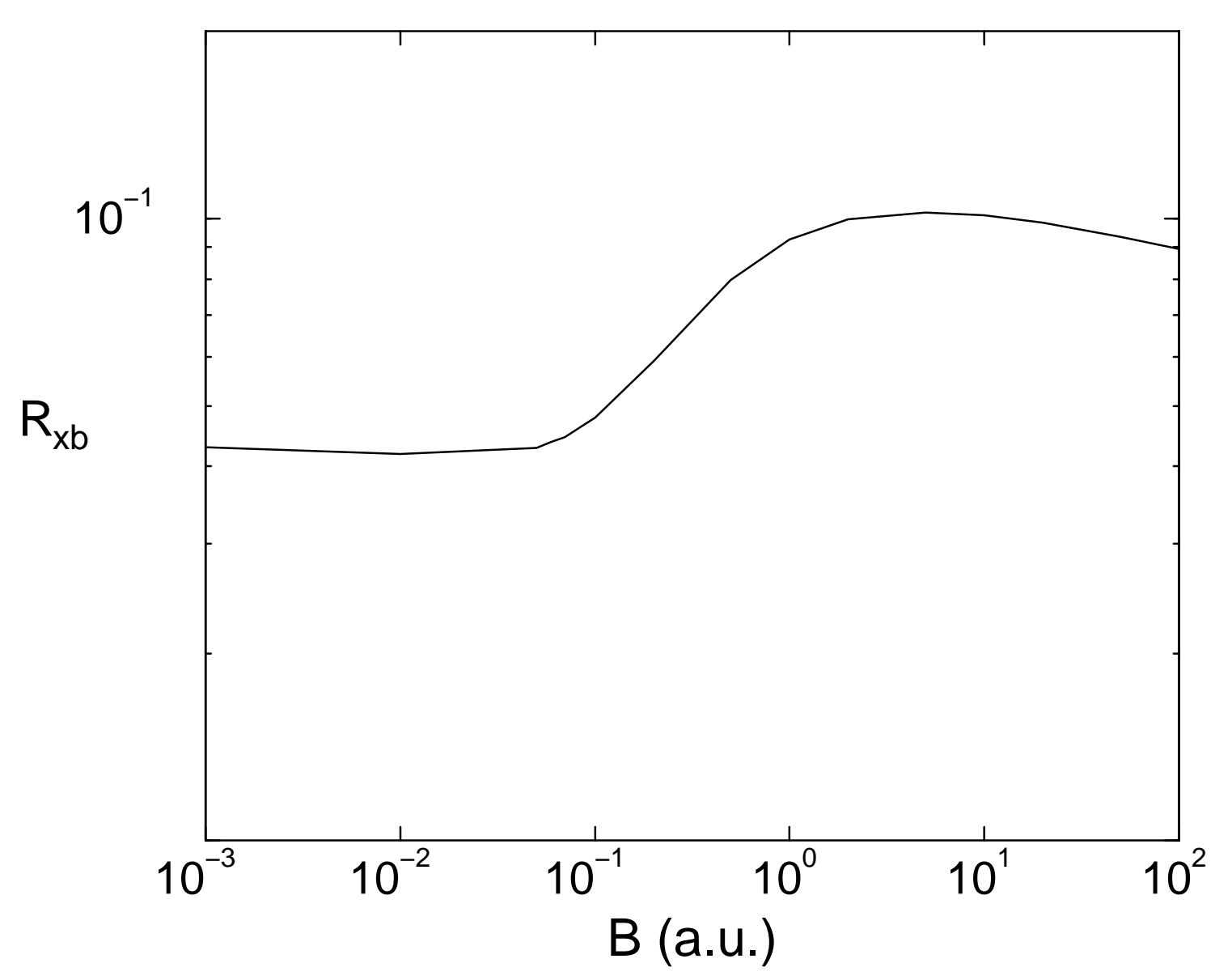


Figure 4

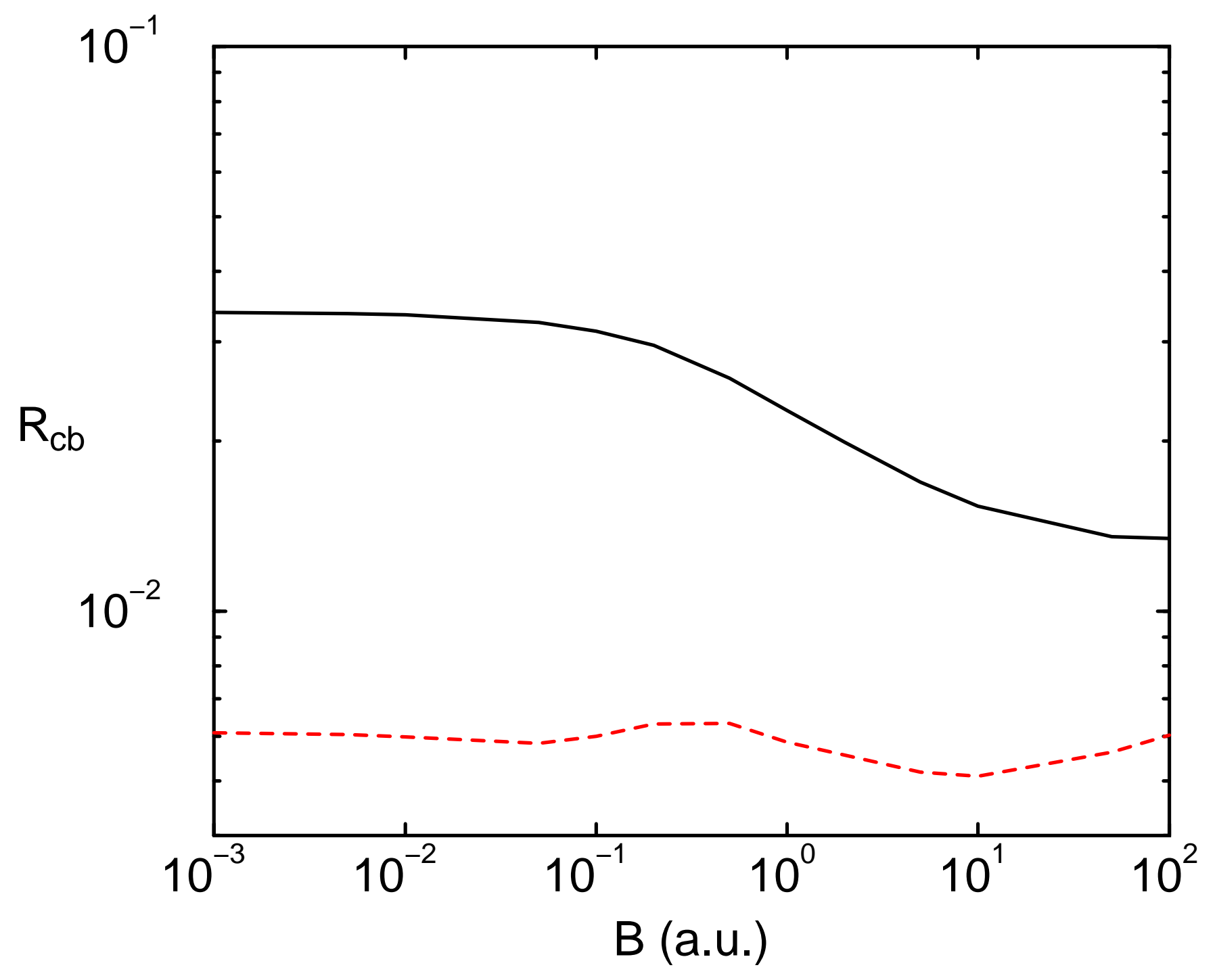


Table1

\begin{tabular}{|c||c|c|c|c|}
\hline $\mathrm{B}$ & $E_{e x}\left(\mathrm{He}\left({ }^{3}(-1)^{+}\right)\right)$ & $E_{e x}\left(\operatorname{Li}\left({ }^{2} 0^{+}\right)\right)$ & $E_{e x}\left(\operatorname{Li}\left({ }^{2}(-1)^{+}\right)\right)$ & $E_{e x}\left(\operatorname{Li}\left({ }^{4}(-3)^{+}\right)\right)$ \\
\hline \hline 0.0 & 0.00644 & 0.02802 & 0.00596 & 0.05521 \\
\hline 0.0008 & 0.00644 & 0.02802 & 0.00596 & 0.05521 \\
\hline 0.0004 & 0.00645 & 0.02803 & 0.00597 & 0.05532 \\
\hline 0.008 & 0.00646 & 0.02805 & 0.00598 & 0.05566 \\
\hline 0.02 & 0.00657 & 0.02818 & 0.00609 & 0.05773 \\
\hline 0.04 & 0.00693 & 0.02864 & 0.00645 & 0.06329 \\
\hline 0.08 & 0.00810 & 0.03024 & 0.00764 & 0.07722 \\
\hline 0.16 & 0.01134 & 0.03484 & 0.01100 & 0.10417 \\
\hline 0.24 & 0.01512 & 0.03985 & 0.01510 & 0.12660 \\
\hline 0.4 & 0.02332 & 0.04826 & 0.02464 & 0.16298 \\
\hline 0.5 & 0.02852 & 0.05169 & 0.03118 & 0.18254 \\
\hline 0.8 & 0.04342 & 0.05360 & 0.05207 & 0.23410 \\
\hline 1.0 & 0.05245 & 0.05055 & 0.06625 & 0.26495 \\
\hline 1.6 & 0.07520 & 0.03900 & 0.10682 & 0.34751 \\
\hline 2.0 & 0.08751 & 0.03366 & 0.13127 & 0.39637 \\
\hline 5.0 & 0.14593 & 0.02159 & 0.26145 & 0.66587 \\
\hline 10. & 0.19818 & 0.01885 & 0.38441 & 0.93573 \\
\hline 20. & 0.25867 & 0.01795 & 0.52663 & 1.25801 \\
\hline 50. & 0.35379 & 0.01776 & 0.74930 & 1.77322 \\
\hline 100. & 0.43872 & 0.01793 & 0.94873 & 2.24050 \\
\hline
\end{tabular}

Table2

\begin{tabular}{|c||c|c|}
\hline $\mathrm{B}$ & $E_{\text {cor }}\left(\mathrm{He}\left({ }^{1} 0^{+}\right)\right)$ & $E_{\text {cor }}\left(\mathrm{He}\left({ }^{3}(-1)^{+}\right)\right)$ \\
\hline \hline 0.0000 & 0.041 & 0.0017 \\
\hline 0.0008 & 0.041 & 0.0017 \\
\hline 0.008 & 0.041 & 0.0017 \\
\hline 0.08 & 0.041 & 0.0020 \\
\hline 0.8 & 0.041 & 0.0054 \\
\hline 2.0 & 0.041 & 0.0068 \\
\hline 5.0 & 0.042 & 0.0082 \\
\hline 10.0 & 0.046 & 0.0100 \\
\hline 20.0 & 0.053 & 0.0128 \\
\hline 50.0 & 0.068 & 0.0196 \\
\hline 100.0 & 0.086 & 0.0281 \\
\hline
\end{tabular}


Table3

\begin{tabular}{|c||c|c||c|c|}
\hline $\mathrm{B}$ & $R_{e q}$ & $E_{e x}\left(H_{2}\left({ }^{3} \Sigma_{u}\right)\right)$ & $R_{e q}$ & $E_{e x}\left(H_{2}\left({ }^{3} \Pi_{u}\right)\right)$ \\
\hline \hline 0.000 & 7.9 & 0.4985 & 1.96 & 0.0313 \\
\hline 0.001 & 7.9 & 0.4984 & 1.96 & 0.0315 \\
\hline 0.005 & 7.9 & 0.4985 & 1.96 & 0.0312 \\
\hline 0.01 & 7.9 & 0.4984 & 1.96 & 0.0313 \\
\hline 0.05 & 7.9 & 0.4993 & 1.95 & 0.0343 \\
\hline 0.1 & 7.8 & 0.5007 & 1.95 & 0.0412 \\
\hline 0.2 & 7.7 & 0.5092 & 1.91 & 0.0567 \\
\hline 0.5 & 7.4 & 0.5515 & 1.79 & 0.0951 \\
\hline 1.0 & 7.5 & 0.6396 & 1.59 & 0.1366 \\
\hline 2.0 & - & - & 1.35 & 0.1874 \\
\hline 5.0 & - & - & 1.03 & 0.2705 \\
\hline 10.0 & - & - & 0.82 & 0.3492 \\
\hline 20.0 & - & - & 0.65 & 0.4429 \\
\hline 50.0 & - & - & 0.48 & 0.5938 \\
\hline 100. & - & - & 0.38 & 0.7320 \\
\hline
\end{tabular}

Table4

\begin{tabular}{|c||c|c||c|c||c|c|}
\hline $\mathrm{B}$ & $R_{e q}$ & $E_{c o r}\left(H_{2}\left({ }^{1} \Sigma_{g}\right)\right)$ & $R_{e q}$ & $E_{c o r}\left(H_{2}\left({ }^{3} \Sigma_{u}\right)\right)$ & $R_{e q}$ & $E_{c o r}\left(H_{2}\left({ }^{3} \Pi_{u}\right)\right)$ \\
\hline \hline 0.000 & 1.40 & 0.0397 & 7.9 & 0.00003 & 1.96 & 0.0045 \\
\hline 0.001 & 1.40 & 0.0397 & 7.9 & 0.00003 & 1.96 & 0.0045 \\
\hline 0.005 & 1.40 & 0.0397 & 7.9 & 0.00004 & 1.96 & 0.0045 \\
\hline 0.01 & 1.40 & 0.0397 & 7.9 & 0.00006 & 1.96 & 0.0045 \\
\hline 0.05 & 1.40 & 0.0397 & 7.9 & 0.00006 & 1.95 & 0.0047 \\
\hline 0.1 & 1.39 & 0.0398 & 7.8 & 0.00003 & 1.95 & 0.0052 \\
\hline 0.2 & 1.39 & 0.0402 & 7.7 & 0.00003 & 1.91 & 0.0061 \\
\hline 0.5 & 1.33 & 0.0411 & 7.4 & 0.00003 & 1.79 & 0.0076 \\
\hline 1.0 & 1.24 & 0.0429 & 7.5 & 0.00002 & 1.59 & 0.0087 \\
\hline 2.0 & 1.09 & 0.0465 & - & - & 1.35 & 0.0105 \\
\hline 5.0 & 0.86 & 0.0541 & - & - & 1.03 & 0.0138 \\
\hline 10.0 & 0.70 & 0.0631 & - & - & 0.82 & 0.0177 \\
\hline 50.0 & 0.42 & 0.1004 & - & - & 0.48 & 0.0359 \\
\hline 100. & 0.33 & 0.1277 & - & - & 0.38 & 0.0497 \\
\hline
\end{tabular}

